Published in ApJ LetTers

Preprint typeset using LTEX style emulateapj v. 12/14/05

\title{
CONFIRMATION OF ENHANCED DWARF-SENSITIVE ABSORPTION FEATURES IN THE SPECTRA OF MASSIVE ELLIPTICAL GALAXIES: FURTHER EVIDENCE FOR A NON-UNIVERSAL INITIAL MASS FUNCTION
}

\author{
Pieter G. VAN DOKKUM ${ }^{1}$ AND CHARLIE CONROY ${ }^{2}$ \\ Published in ApJ Letters
}

\begin{abstract}
We recently found that massive cluster elliptical galaxies have strong $\mathrm{Na}$ I $\lambda 8183,8195$ and $\mathrm{FeH} \lambda 9916$ WingFord band absorption, indicating the presence of a very large population of stars with masses $\lesssim 0.3 \mathrm{M}_{\odot}$. Here we test this result by comparing the elliptical galaxy spectra to those of luminous globular clusters associated with M31. These globular clusters have similar metallicities, abundance ratios and ages as massive elliptical galaxies but their low dynamical mass-to-light ratios rule out steep stellar initial mass functions (IMFs). From high quality Keck spectra we find that the dwarf-sensitive absorption lines in globular clusters are significantly weaker than in elliptical galaxies, and consistent with normal IMFs. The differences in the Na I and Wing-Ford indices are $0.027 \pm 0.007 \mathrm{mag}$ and $0.017 \pm 0.006$ mag respectively. We directly compare the two classes of objects by subtracting the averaged globular cluster spectrum from the averaged elliptical galaxy spectrum. The difference spectrum is well fit by the difference between a stellar population synthesis model with a bottomheavy IMF and one with a bottom-light IMF. We speculate that the slope of the IMF may vary with velocity dispersion, although it is not yet clear what physical mechanism would be responsible for such a relation.
\end{abstract}

Subject headings: cosmology: observations — galaxies: abundances — galaxies: evolution — galaxies: stellar content - stars: mass function

\section{INTRODUCTION}

The form of the stellar initial mass function (IMF) is one of the main uncertainties in the interpretation of observations of distant galaxies and in our understanding of the conversion of gas to stars over cosmic time. Most of the stellar mass density of the Universe is in the form of low mass stars with masses $\ll 1 \mathrm{M}_{\odot}$. Despite the large contribution of these stars to the total stellar mass of galaxies their contribution to the integrated light is small, which makes it difficult to measure the form of the IMF in galaxies other than the Milky Way. In practise it is usually assumed that the IMF in other galaxies and at earlier epochs is the same as in the disk of the Milky Way: a power-law with logarithmic slope $x=-2.3$ at masses $\gtrsim 1 \mathrm{M}_{\odot}$ with a turnover at lower masses (Kroupa 2001; Chabrier 2003; Bastian, Covey, \& Meyer 2010).

As has long been recognized it is possible to measure the contribution of dwarf stars to the integrated light of old stellar populations from absorption features that have a strong gravity dependence (e.g., Spinrad 1962; Cohen 1978; Faber $\&$ French 1980). There are two absorption features in the red that are strong in cool dwarfs but very weak or absent in cool giants: the Na I $\lambda 8183,8195 \AA$ doublet (e.g., Schiavon et al. 1997) and the Wing-Ford FeH molecular band at $\lambda 9916 \AA$ (e.g., Wing \& Ford 1969; Schiavon, Barbuy, \& Bruzual A. 2000). These features reach depths of $30-40 \%$ and $40-50 \%$ respectively in the spectra of individual low mass stars. In the integrated light of old stellar populations their predicted strengths are of order 1-5\% depending on the form of the IMF (see van Dokkum \& Conroy 2010, herafter vDC).

In vDC we presented high quality Keck spectra for eight massive galaxies in the Coma and Virgo clusters. Both the $\mathrm{Na}$ I doublet and the Wing-Ford band are quite strong in these spectra, in agreement with previous studies (e.g., Carter, Vis-

\footnotetext{
${ }^{1}$ Department of Astronomy, Yale University, New Haven, CT 06520 , USA

${ }^{2}$ Harvard-Smithsonian Center for Astrophysics, 60 Garden Street, Cambridge, MA 02138, USA
}

vanathan, \& Pickles 1986; Hardy \& Couture 1988; Couture \& Hardy 1993). As shown in vDC, the absorption is significantly stronger (by a factor of $\sim 3$ ) than might be expected from a $10 \mathrm{Gyr}$ old Solar metallicity stellar population with a Milky-Way IMF. The best-fitting IMF is more dwarf-enriched than even the Salpeter (1955) form, requiring a slope $x \sim-3$ down to $0.1 \mathrm{M}_{\odot}$ (compared to $x=-2.35$ for a Salpeter IMF).

The main uncertainty in vDC is that we rely on stellar population synthesis models. The models are based on highquality empirical spectra of individual Solar metallicity stars from the Rayner, Cushing, \& Vacca (2009) IRTF library of cool stars, and use up-to-date isochrones (see Conroy, Gunn, \& White 2009, vDC, and C. Conroy et al., in preparation). However, it is known that the stars in elliptical galaxies are enhanced in $\alpha$-elements compared to stars in the Milky Way (Worthey, Faber, \& Gonzalez 1992) and this cannot be incorporated explicitly in our modeling.

In this Letter we test whether the enhancement of dwarfsensitive spectral features in elliptical galaxies persists when we compare their spectra to those of globular clusters rather than to models. The Andromeda galaxy (M31) has a population of old, metal-rich globular that have similar abundance patterns as massive elliptical galaxies (Caldwell et al. 2011). However, their dynamical mass-to-light $(M / L)$ ratios are so low that they must have normal mass functions - or possibly even mass functions that are dwarf-deficient ("bottom-light") compared to the Milky Way IMF (Strader et al. 2011). Therefore, if the interpretation in vDC is correct, we should see much weaker $\mathrm{Na}$ I and $\mathrm{FeH}$ absorption than in massive elliptical galaxies, despite the fact that their ages and abundances are very similar.

\section{SAMPLE SELECTION AND OBSERVATIONS}

We observed four bright globular clusters associated with M31 for which ages and metallicities had been determined in other studies: B143, B147, B163, and B193. The clusters were selected to be bright and old and to have approximately Solar iron abundances (Caldwell et al. 2011). For a 


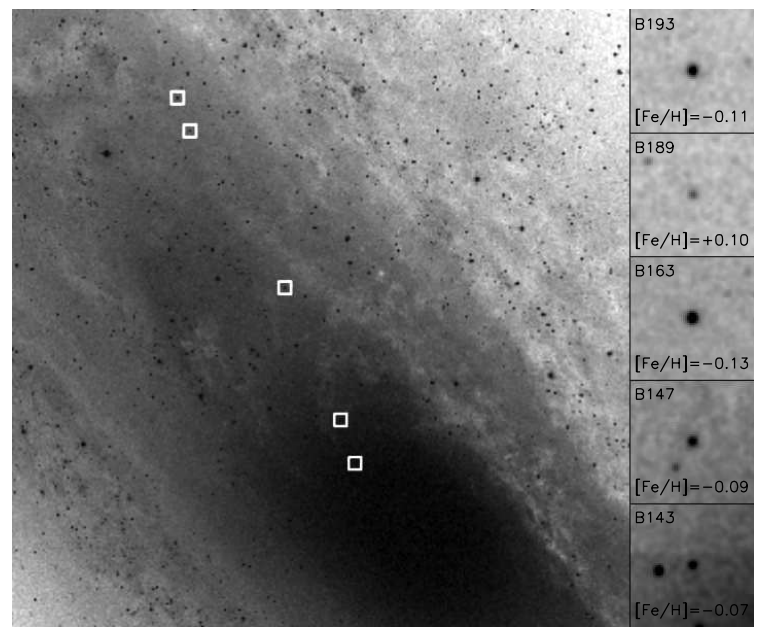

FIG. 1.- The five globular clusters that were observed with LRIS. The main image is an optical image from the Digital Sky Survey and the insets are $J$ band images from 2MASS. Four of the clusters are among the brightest in M31 and have approximately Solar metallicity (Caldwell et al. 2011). The relatively faint cluster B189 was added to the sample to test how sensitive the conclusions are to abundance patterns.

Chabrier (2003) IMF the total number of stars in these four clusters is approximately $10^{7}$ (of which $\sim 100$ should be AGB stars), which is sufficient to properly sample the stellar luminosity function. We also observed the very metal-rich cluster B189 to assess the sensitivity of the $\mathrm{FeH}$ and $\mathrm{NaI}$ features to changes in abundance patterns (see below). The locations of the clusters within M31 are indicated in Fig. 11 In Fig. 2 their abundances and ages are compared to those of the elliptical galaxies of vDC. The globular cluster data come from Caldwell et al. (2011) and R. Schiavon et al., in prep. Data for the four Virgo galaxies were obtained from Trager et al. (2000), for an $r_{e} / 8$ aperture (which is similar to the effective aperture of our measurements of Virgo galaxies). The Coma measurements are from Thomas et al. (2005) (NGC 4840 and NGC 4926) and from Harrison et al. (2010) (NGC 4889 and IC 3976). All measurements should be on approximately the same system, although systematic differences between studies cannot be ruled out. The four luminous M31 globular clusters have similar ages and abundances as the elliptical galaxies. The cluster B189 has a higher iron abundance and $\alpha$-enhancement than most of the ellipticals. Furthermore, it is extremely enhanced in $\mathrm{C}$ and $\mathrm{N}$, and it has strong $\mathrm{NaD}$ absorption (R. Schiavon, priv. comm.).

The globular clusters were observed on December 3, 2010 with the red arm of the Low Resolution and Imaging Spectrometer (LRIS; Oke et al. 1995) on the Keck I telescope. We used the $6001 \mathrm{~mm}^{-1}$ grating blazed at $1 \mu \mathrm{m}$. The slit width of $0{ }^{\prime \prime} 7$ gives a spectral resolution of $\sigma=1.2 \AA$ at $9500 \AA$, corresponding to a velocity resolution of $\approx 40 \mathrm{~km} \mathrm{~s}^{-1}$. Each cluster was dithered along the slit in a series of four $300 \mathrm{~s}$ exposures. Data reduction followed standard procedures for longslit spectroscopy. Owing to the fully-depleted LBNL CCDs of LRIS fringing is not a concern. We corrected for atmospheric absorption in the following way. Before and after each globular cluster we observed the Galactic A star HIP 2860. Next, we convolved a high S/N Mauna Kea night sky spectrum to the instrumental resolution and scaled the absorption to match strong observed atmospheric absorption lines in HIP 2860. Finally, for each globular cluster we interpolated the two scaled

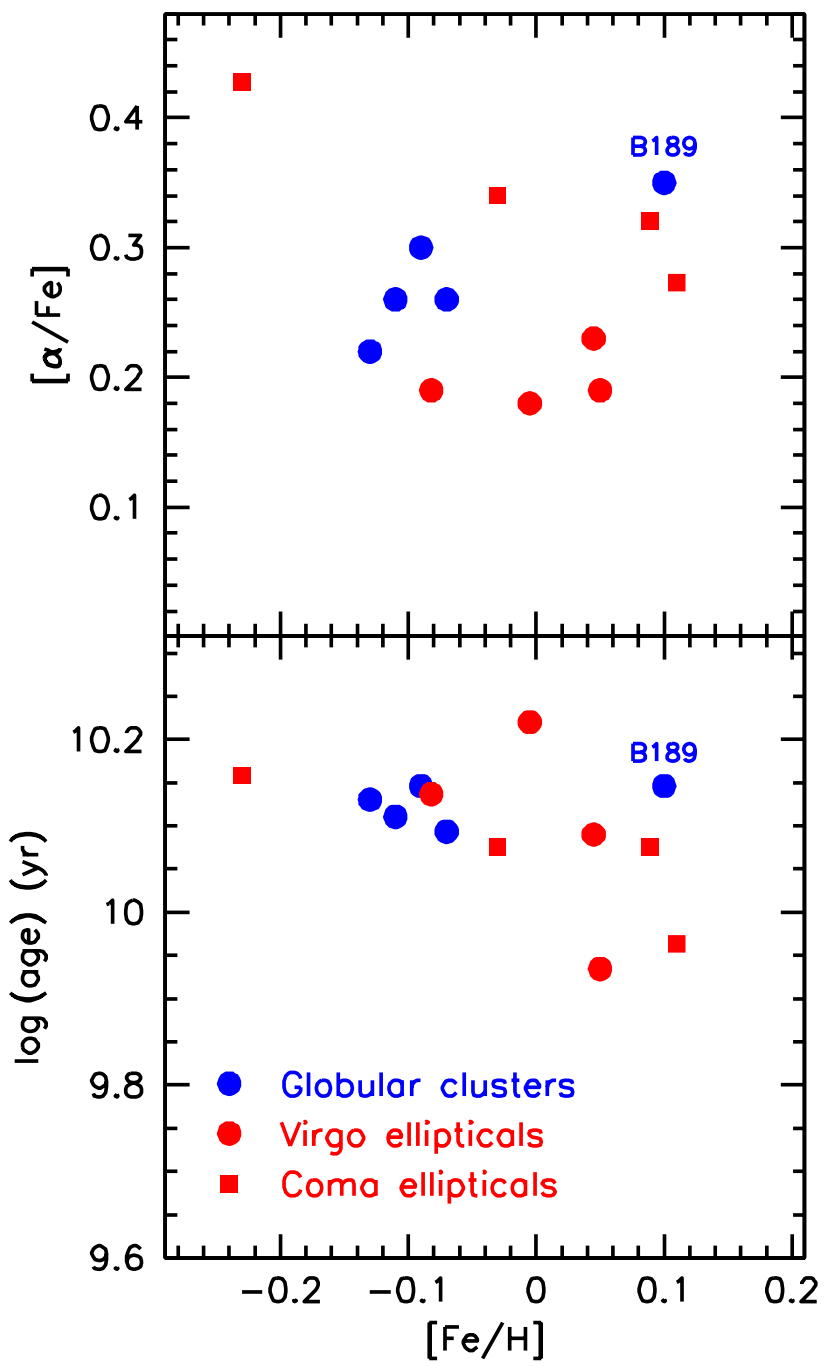

FIG. 2.- Comparison of the iron abundances $[\mathrm{Fe} / \mathrm{H}], \alpha$ enhancement $[\alpha / \mathrm{Fe}]$, and ages of the M31 globular clusters to those of the elliptical galaxies of vDC. The M31 clusters have similar abundance patterns and ages as the elliptical galaxies. Typical errors in individual measurements are $0.1-0.2$ dex, i.e., most of the data points are within $1 \sigma$ of one another.

absorption spectra obtained from the HIP 2860 observations bracketing that cluster. This procedure worked well, leaving no detectable residuals of night sky absorption lines in the wavelength regions of interest.

One-dimensional spectra were extracted by summing the central 13 rows of the two-dimensional spectra, corresponding to $1 . " 8$ (7 pc, which is approximately the half-light diameter of the clusters). The results are independent of the extraction aperture; as expected (see §5) we are not able to detect mass segregation in these clusters. The spectra of the four most luminous globular clusters were de-blueshifted to restframe wavelengths, normalized, and averaged in order to increase the $\mathrm{S} / \mathrm{N}$ ratio and diminish the effects of any remaining systematic detector or night sky residuals. The averaging of these four clusters is justified as their ages and metallicities are nearly identical (see Fig. 2). In the following the cluster B189 will be discussed separately, although we note here that including this cluster in the average spectrum has no impact on our analysis or conclusions. 

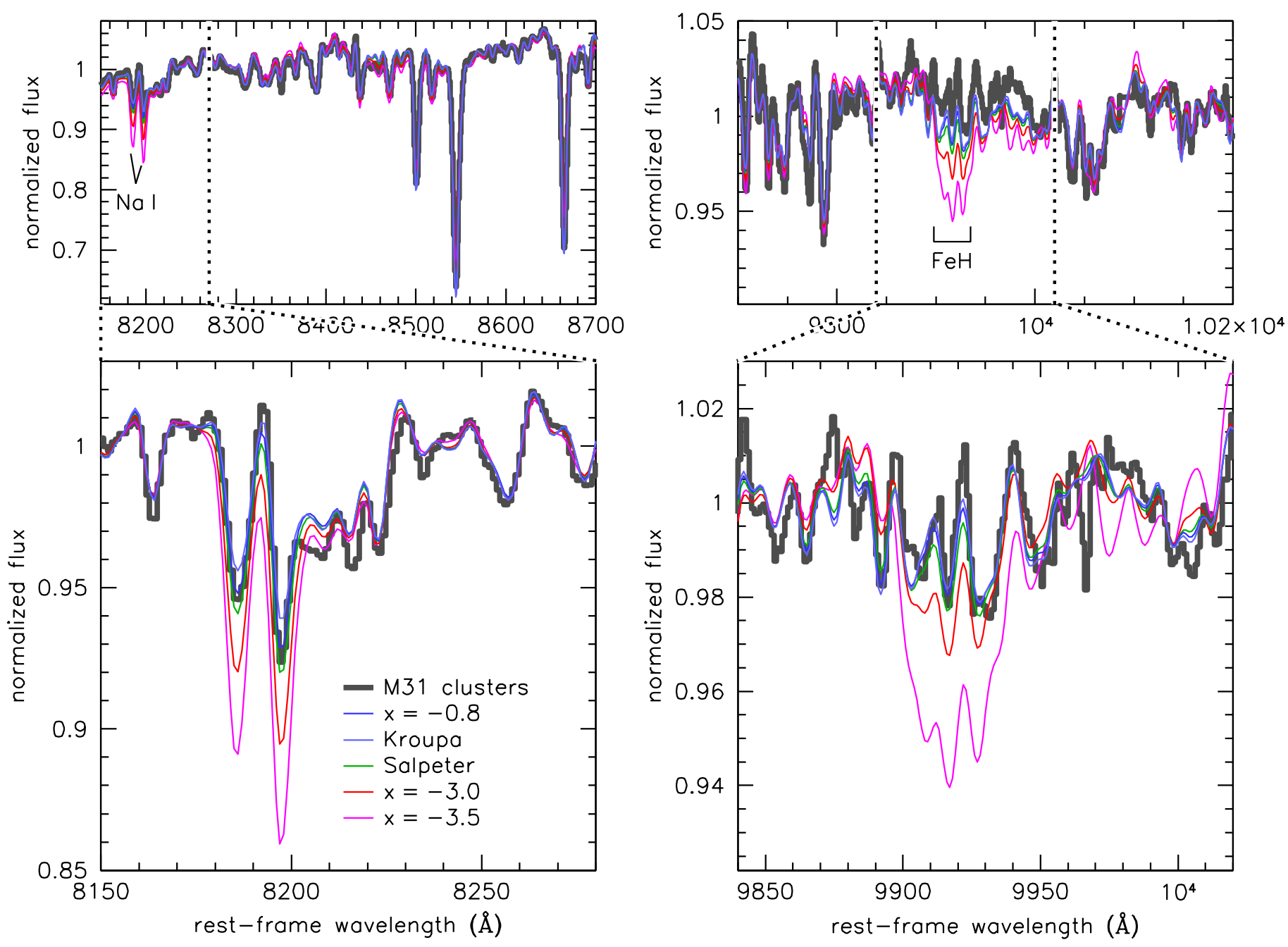

FIG. 3.- Averaged Keck spectra of the M31 globular clusters B143, B147, B163, and B193 (thick grey lines). The left panels show the region near the Na I doublet and the right panels show the FeH Wing-Ford band region. The bottom panels zoom in on the IMF sensitive features. Colored lines show predictions from stellar population synthesis models with different IMFs. Models with "normal" IMFs match the data very well over the entire wavelength range. Dwarf-rich IMFs with steep slopes $(x \sim-3)$ — which provide good fits to massive elliptical galaxies do not fit the observed weak Na I doublet and Wing-Ford band of the globular clusters.

\section{THE NA I DOUBLET AND THE WING-FORD BAND IN M31 GLOBULAR CLUSTERS}

Figure 3 shows the averaged spectrum of the M31 globular clusters in the wavelength regions near the $\mathrm{NaI}$ doublet (left panel) and the Wing-Ford band (right panel). Note that the $\mathrm{Na}$ I doublet is resolved and not blended with $\mathrm{TiO}$, as globular clusters have much lower velocity dispersions than the elliptical galaxies discussed in vDC. Following vDC the spectrum was normalized by fitting second-order polynomials in the top panels (excluding the regions around the features of interest) and first-order polynomials in the bottom panels. The typical uncertainty in the averaged spectrum is $0.003 \AA^{-1}$, as judged from the median scatter among the four globular clusters over the wavelength range $8200 \AA-8400 \AA$. All the easily visible absorption lines are stellar features, not noise. We smoothed the spectra slightly to approximate the resolution of the IRTF spectral library (see below).

Colored lines are stellar population synthesis models. The models are the same as those in vDC: they are 10 Gyr old, Solar metallicity models based on empirical stellar spectra from the Rayner et al. (2009) IRTF library. Models with slightly different ages are virtually identical. The models will be presented in detail in C. Conroy et al., in preparation. Different colors indicate different dwarf contributions, ranging from bottom-light to bottom-heavy IMFs. The data are well fit by models with a Kroupa (2001) or bottom-light IMF: the median absolute deviation is $0.005 \AA^{-1}$ from $8150 \AA-8700 \AA$ and $0.007 \AA^{-1}$ from $9700 \AA-10,200 \AA$. This demonstrates that our stellar population synthesis model, which is based on Solar metallicity stars in the Milky Way, provides an adequate description of the spectra of globular clusters in M31.

We have shown in vDC that elliptical galaxies are best fit by a bottom-heavy IMF with $x=-3$ (the red line in Fig. 3). The globular clusters are poorly fit by such models. In the $\mathrm{NaI}$ band ( $8185 \AA-9205 \AA$; see vDC) the median absolute deviation is $0.025 \pm 0.005$ for the $x=-3 \mathrm{IMF}$, compared to 0.004 for a Kroupa (2001) IMF. In the Wing-Ford band (9910 $9930 \AA$ ) the median absolute deviation is $0.015 \pm 0.005$ for $x=-3$ and 0.003 for Kroupa. 

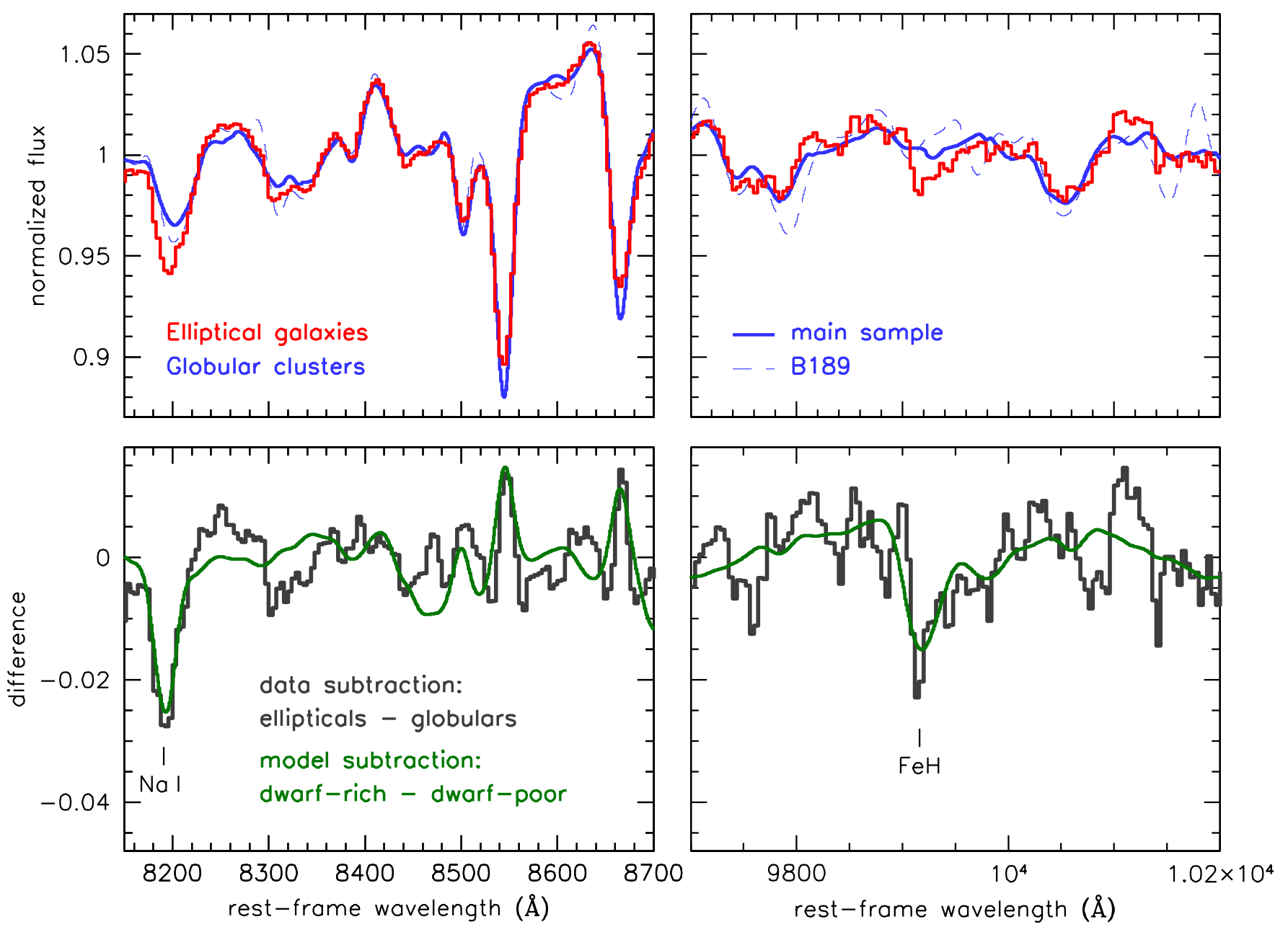

FIG. 4.- Top panels: Direct comparison of the globular cluster spectra to the elliptical galaxy spectra. The globular cluster spectra were smoothed to match the average velocity dispersion of the elliptical galaxies. The main sample comprises the luminous clusters B143, B147, B163 and B193. The cluster B189 is shown separately; note that the S/N ratio of this spectrum is much lower than that of the averaged spectrum of the other clusters. Bottom panels: The black spectrum is the difference between the elliptical galaxies and the main sample of globular clusters. The Doppler-broadened Na I doublet and the Wing-Ford band are clearly much stronger in the elliptical galaxies than in the globular clusters. The green line is the difference between a stellar population synthesis model with an enhanced dwarf population $(x=-3)$ and one with a depressed dwarf population $(x=-0.8)$. The green line is a good fit to the black line, which shows that IMF variations can explain the spectral differences between globular clusters and massive elliptical galaxies.

\section{DIRECT COMPARISON BETWEEN GLOBULAR CLUSTERS AND ELLIPTICAL GALAXIES}

The fact that stellar population synthesis models indicate a normal IMF for globular clusters is significant as those same models indicated a bottom-heavy IMF for elliptical galaxies. We can also compare the globular clusters to the elliptical galaxies in a more direct way. In the top panels of Fig. 4 we show the averaged cluster spectrum along with the averaged elliptical galaxy spectrum. The Coma and Virgo spectra of vDC were averaged, and the globular cluster spectrum was smoothed to the same velocity width as the elliptical galaxies. As expected, the averaged globular cluster spectrum is very similar to the averaged elliptical galaxy spectrum, except in the regions around $\mathrm{NaI}$ and the Wing-Ford band. The globular cluster B189 is shown separately. Although the absorption lines in this cluster are slightly stronger than in the other clusters owing to its higher metallicity, the NaI and WingFord band do not reach the depth seen in the ellipticals. This demonstrates that our conclusions are insensitive to residual metallicity or abundance variations between globular clusters and elliptical galaxies.

The bottom panels of Fig. 4 show the difference spectrum, obtained by subtracting the averaged spectrum of the globular clusters from that of the elliptical galaxies. The two most significant features in the difference spectrum are at $\sim 8190 \AA$ and at $\sim 9920 \AA$. In both cases the absorption in the ellipticals is much stronger than in the globular clusters. The wavelengths of these features correspond to those of the Dopplerbroadened $\mathrm{Na}$ I doublet and the Wing-Ford band respectively, demonstrating empirically that these dwarf-sensitive features are significantly stronger in massive elliptical galaxies than in M31 globular clusters. Expressed as an index difference, the $\mathrm{NaI}$ index (as defined in vDC) is $0.027 \pm 0.007$ mag stronger and the Wing-Ford index is $0.017 \pm 0.006$ mag stronger, where the uncertainties are determined from the scatter among the individual objects entering the two spectra.

The green line in Fig. 4 shows the difference between two of the models shown in Fig. 3, a model with a bottom-heavy IMF ( $x=-3$, which was the best fit to the elliptical galaxies in vDC) and a model with a bottom-light IMF $(x=-0.8$, 
which provides a good fit to the $M / L$ ratios of globular clusters [Strader et al. 2011]). The models have identical age (10 Gyr) and Solar metallicity; the only difference is the number of low mass stars. The green line is a good fit to the difference spectrum, as might have been expected from the analyses in vDC and $\S 3$. The model also reproduces the fact that the $\mathrm{Ca}$ II $\lambda 8498, \lambda 8542$, and $\lambda 8662$ lines are weaker in the globular clusters; in the stellar population synthesis model this reflects the weak Ca II absorption in dwarfs compared to giants. Although giants dominate the light, for an IMF as steep as $x=-3$ there are sufficient dwarfs to slightly depress the $\mathrm{Ca}$ lines. The relative weakness of the $\mathrm{Ca}$ triplet in elliptical galaxies was previously discussed by, e.g., Cenarro et al. (2003). We note, however, that in our differential analysis this effect is very sensitive to small errors in matching the velocity dispersions of the two spectra.

\section{DISCUSSION}

We have shown that massive cluster elliptical galaxies have much stronger $\mathrm{NaI}$ and $\mathrm{FeH}$ absorption than old metal-rich globular clusters in M31. This constitutes a critical test of the analysis in vDC: equally strong absorption in the globular clusters would have implied that the enhancement in elliptical galaxies had incorrectly been interpreted as an IMF effect, as the low $M / L$ ratios of globular clusters are inconsistent with dwarf-rich IMFs. Although we cannot exclude that some unknown stellar population effect is responsible for the strong absorption in elliptical galaxies, it is striking how well the difference spectrum in Fig. 4 is fit by an enhanced dwarf population. Any explanation not involving dwarf stars has to account for the enhanced $\mathrm{NaI}$ and $\mathrm{FeH}$ absorption in ellipticals, the suppressed $\mathrm{Ca}$ II, and the similarity of $\mathrm{TiO}, \mathrm{Fe}$, $\mathrm{Mg}, \mathrm{H} \beta$ and other elements. We note that known abundance anomalies in Galactic globular clusters work in the opposite direction (Gratton, Sneden, \& Carretta 2004).

If dwarfs are indeed the cause of the differences in absorption then Fig. 4 constitutes direct evidence for significant variation in the low mass IMF. Quantifying the IMF in elliptical galaxies using stellar population synthesis models, we can confidently rule out dwarf-suppressed IMFs such as those proposed by van Dokkum (2008) and Davé (2008) and also "standard" Kroupa (2001) or Chabrier (2003) IMFs which are appropriate for the Milky Way disk. This conclusion does not depend on the detailed present-day stellar mass function of globular clusters (which may have been modified by dynamical effects), as the expected strengths of the $\mathrm{NaI}$ line and the Wing-Ford band are almost identical for all "light" IMFs (Schiavon et al. 2000; Fig. 3).

Star formation likely proceeded very differently in massive elliptical galaxies than in the disks of spiral galaxies. It is now thought that the progenitors of massive ellipticals were very compact, with average densities $\gtrsim 20 \mathrm{M}_{\odot} \mathrm{pc}^{-3}$ inside the effective radius (e.g., van Dokkum et al. 2008; Buitrago et al. 2008). These densities are similar to giant molecular clouds in the Milky Way, but given the $\sim$ kpc scale of these galaxies the column densities would have been several orders of magnitude higher. It will be interesting to measure the physical conditions in the star-forming progenitors of these galaxies and compare them to measurements in star-forming regions in the Milky Way (see, e.g., Ivison et al. 2010).

This study can be extended in many ways. The comparison between globular clusters and elliptical galaxies itself is model-independent, but we still rely on stellar population synthesis models to quantify how steep the IMF is in elliptical galaxies. The main uncertainty in these models is whether short-lived stellar evolution phases with unusual abundance patterns are missed, and this can be addressed by augmenting the spectral library. Radial gradients in $\mathrm{NaI}$ and $\mathrm{FeH}$ can provide information on the spatial distribution of the dwarfs, which in turn might reflect different formation mechanisms for the central of ellipticals and their outskirts (e.g., Oser et al. 2010; van Dokkum et al. 2010). Some studies find steep gradients in Na I (Boroson \& Thompson 1991), which might suggest that such effects could be important. It will also be interesting to extend this study to elliptical galaxies of lower luminosity. There is good evidence that the dynamical $M / L$ ratio of early-type systems scales with velocity dispersion (e.g., Cappellari et al. 2006; Forbes et al. 2008; Treu et al. 2010). A varying IMF may be responsible for this trend (Treu et al. 2010, Dutton et al. 2010), although some studies consider dark matter variations a more likely possibility (Graves \& Faber 2010). As noted by Cappellari et al. (2006) the dynamical $M / L$ ratios of low luminosity ellipticals may require a normal IMF, as they appear to be lower than the $M / L$ ratio of a stellar population with a bottom-heavy IMF $\left(M / L_{B} \approx 23\right.$ and $M / L_{R} \approx 13$ for $\left.x=-3\right)$. A preliminary analysis of a Keck spectrum of the elliptical galaxy NGC 4458 $\left(\sigma=85 \mathrm{~km} \mathrm{~s}^{-1}\right.$; Cappellari et al. 2006) shows that its dwarfsensitive features are indeed similar to those of the M31 globular clusters. Finally, it will be interesting to re-examine studies of the evolution of scaling relations of early-type galaxies (e.g., Renzini 2006). The color and $M / L$ evolution of massive galaxies in clusters seem to require a bottom-light IMF with $x=-0.7_{-0.3}^{+0.7}$ (van Dokkum 2008), but it may be possible to fit the data with steeper mass functions when structural evolution is incorporated in the modeling (e.g., van der Wel et al. 2008; van Dokkum 2008; Hopkins et al. 2009; Holden et al. 2010).

A varying IMF has important implications for the interpretation of observations of distant galaxies. The $M / L$ ratios of stellar populations with an $x=-3$ IMF and a Kroupa IMF differ by approximately a factor of 3-4. If it is not known how the IMF varies with galaxy type, this uncertainty directly corresponds to the systematic uncertainty in the stellar masses and star formation rates of galaxies. Even though massive elliptical galaxies make up only a small fraction of the galaxy population today, their old ages imply that their progenitors become increasingly more dominant at higher redshifts. As a result, the star formation history of the Universe, the evolution of the galaxy mass function, and many other observations would have to be revised.

We are grateful to Ricardo Schiavon and Nelson Caldwell for providing metallicities and ages of M31 globular clusters prior to publication. We thank Judy Cohen, Richard Larson, Ricardo Schiavon, and Scott Trager for useful discussions.

\section{REFERENCES}

Bastian, N., Covey, K. R., \& Meyer, M. R. 2010, ARA\&A, 48, 339

Boroson, T. A. \& Thompson, I. B. 1991, AJ, 101, 111

Buitrago, F., Trujillo, I., Conselice, C. J., Bouwens, R. J., Dickinson, M., \& Yan, H. 2008, ApJ, 687, L61
Caldwell, N., Schiavon, R., Morrison, H., Rose, J. A., \& Harding, P. 2011, AJ, 141, 61

Cappellari, M., Bacon, R., Bureau, M., Damen, M. C., Davies, R. L., de Zeeuw, P. T., Emsellem, E., Falcón-Barroso, J., et al. 2006, MNRAS, 366, 1126 
Carter, D. Visvanathan, N. \& Pickles, A. J. 1986, ApJ, 311, 637

Cenarro, A. J., Gorgas, J., Vazdekis, A., Cardiel, N., \& Peletier, R. F. 2003 , MNRAS, 339, L12

Chabrier, G. 2003, PASP, 115, 763

Cohen, J. G. 1978, ApJ, 221, 788

Conroy, C., Gunn, J. E., \& White, M. 2009, ApJ, 699, 486

Couture, J. \& Hardy, E. 1993, ApJ, 406, 142

Davé, R. 2008, MNRAS, 385, 147

Dutton, A. A., et al. 2010, MNRAS, submitted (arXiv:1012.5859

Faber, S. M. \& French, H. B. 1980, ApJ, 235, 405

Forbes, D. A., Lasky, P., Graham, A. W., \& Spitler, L. 2008, MNRAS, 389, 1924

Gratton, R., Sneden, C., \& Carretta, E. 2004, ARA\&A, 42, 385

Graves, G. J. \& Faber, S. M. 2010, ApJ, 717, 803

Hardy, E. \& Couture, J. 1988, ApJ, 325, L29

Harrison, C. D., Colless, M., Kuntschner, H., Couch, W. J., de Propris, R., \& Pracy, M. B. 2010, MNRAS, 409, 1455

Holden, B. P., van der Wel, A., Kelson, D. D., Franx, M., \& Illingworth, G. D. 2010, ApJ, 724, 714

Hopkins, P. F., Hernquist, L., Cox, T. J., Keres, D., \& Wuyts, S. 2009, ApJ, 691, 1424

Ivison, R. J., Swinbank, A. M., Swinyard, B., Smail, I., Pearson, C. P. Rigopoulou, D., Polehampton, E., Baluteau, J., et al. 2010, A\&A, 518, L35

Kroupa, P. 2001, MNRAS, 322, 231

Oke, J. B., et al. 1995, PASP, 107, 375
Oser, L., Ostriker, J. P., Naab, T., Johansson, P. H., \& Burkert, A. 2010, ApJ, 725,2312

Rayner, J. T., Cushing, M. C., \& Vacca, W. D. 2009, ApJS, 185, 289

Renzini, A. 2006, ARA\&A, 44, 141

Salpeter, E. E. 1955, ApJ, 121, 161

Schiavon, R. P., Barbuy, B., \& Bruzual A., G. 2000, ApJ, 532, 453

Schiavon, R. P., Barbuy, B., Rossi, S. C. F., \& Milone, A. 1997, ApJ, 479, 902

Spinrad, H. 1962, ApJ, 135, 715

Strader, J., Caldwell, N., \& Seth, A. 2011, AJ, in press (arXiv:1104.4649)

Thomas, D., Maraston, C., Bender, R., \& Mendes de Oliveira, C. 2005, ApJ, 621,673

Trager, S. C., Faber, S. M., Worthey, G., \& González, J. J. 2000, AJ, 119, 1645

Treu, T., Auger, M. W., Koopmans, L. V. E., Gavazzi, R., Marshall, P. J., \& Bolton, A. S. 2010, ApJ, 709, 1195

van der Wel, A., Holden, B. P., Zirm, A. W., Franx, M., Rettura, A., Illingworth, G. D., \& Ford, H. C. 2008, ApJ, 688, 48

van Dokkum, P. G. 2008, ApJ, 674, 29

van Dokkum, P. G. \& Conroy, C. 2010, Nature, 468, 940 [vDC]

van Dokkum, P. G., Franx, M., Kriek, M., Holden, B., Illingworth, G. D., Magee, D., Bouwens, R., Marchesini, D., et al. 2008, ApJ, 677, L5

van Dokkum, P. G., Whitaker, K. E., Brammer, G., Franx, M., Kriek, M., Labbé, I., Marchesini, D., Quadri, R., et al. 2010, ApJ, 709, 1018

Wing, R. F. \& Ford, Jr., W. K. 1969, PASP, 81, 527

Worthey, G., Faber, S. M., \& Gonzalez, J. J. 1992, ApJ, 398, 69 